International Journal of Applied Mathematics

Volume 29 No. $3 \quad 2016,331-347$

ISSN: 1311-1728 (printed version); ISSN: 1314-8060 (on-line version)

doi: http://dx.doi.org/10.12732/ijam.v29i3.5

\title{
THE EXISTENCE AND UNIQUENESS OF POSITIVE WEIGHTED PSEUDO ALMOST AUTOMORPHIC SOLUTION FOR SOME SYSTEMS OF NEUTRAL NONLINEAR DELAY INTEGRAL EQUATIONS
}

\author{
Abdellatif Sadrati ${ }^{1 \S}$, Abderrahim Zertiti ${ }^{2}$ \\ ${ }^{1,2}$ Department of Mathematics \\ Faculty of Sciences \\ University Abdelmalek Essaâdi \\ B.P. 2121, Tetouan, MOROCCO
}

\begin{abstract}
In this paper, we investigate the existence and uniqueness of positive weighted pseudo almost automorphic solution to some systems of neutral nonlinear integral equations with variant delays. Our purpose is to extend some existing results in the scalar case and to generalize others in the case of systems. An example is given to illustrate our results.
\end{abstract}

AMS Subject Classification: 45G10, 45G15, 47H30

Key Words: almost automorphy, weighted pseudo almost automorphic solution, nonlinear delay integral systems, Thompson's part metric, unique fixed point

\section{Introduction}

Let us consider the following system

$$
\begin{aligned}
& x(t)=\gamma_{1}(t) x\left(t-\beta_{1}\right)+\int_{t-\sigma_{1}(t)}^{t} f(s, x(s), y(s)) d s, \\
& y(t)=\gamma_{2}(t) y\left(t-\beta_{2}\right)+\int_{t-\sigma_{2}(t)}^{t} g(s, x(s), y(s)) d s,
\end{aligned}
$$

Received: March 15, 2016

(c) 2016 Academic Publications

${ }^{\S}$ Correspondence author 
where $f, g: \mathbb{R} \times \mathbb{R}^{+} \times \mathbb{R}^{+} \longrightarrow \mathbb{R}, \gamma_{1}, \gamma_{2}: \mathbb{R} \longrightarrow \mathbb{R}$ are nonnegative continuous functions and $\sigma_{1}, \sigma_{2}: \mathbb{R} \longrightarrow \mathbb{R}$ are positive continuous functions. Moreover,

$$
f(t, 0, y)=g(t, x, 0)=0, \forall(t, x, y) \in \mathbb{R} \times \mathbb{R}^{+} \times \mathbb{R}^{+} .
$$

The system (1) with positive solutions describes the evolution in time of two species $x$ and $y$ with interaction. Briefly, $x(t), y(t)$ are, respectively, the numbers of individuals present in the populations $x, y$ at time $t$ and, which live to the ages $\sigma_{1}(t), \sigma_{2}(t)$. Functions $f, g$ are, respectively, the numbers of new births per time unit in $x, y$. Also, we can describe (1) in the context of epidemics. $x(t), y(t)$ are the populations at time $t$ of infectious individuals, $\sigma_{1}(t), \sigma_{2}(t)$ are the durations of infectivity and the functions $f, g$ are the instantaneous rates of infection.

In [9], Cooke and Kaplan initiated the study of the scalar delay integral equation

$$
x(t)=\int_{t-\tau}^{t} f(s, x(s)) d s,
$$

where $f(t, x)$ is a continuous function which is periodic in $t$. The authors in [9] established sufficient conditions for the existence of positive periodic solution of (2). Since then, many works were concerned with the existence of periodic, almost periodic, pseudo almost periodic and almost automorphic solutions for various kinds of $(2)$. We refer the reader to $[1,2,8,10,11]$ and the references therein.

The existence of almost periodic solutions for a scalar version of system (1) in the case of $\sigma(t) \equiv \sigma \in(0,+\infty)$ and $\gamma(t) \equiv \gamma \in[0,1)$

$$
x(t)=\gamma x(t-\sigma)+(1-\gamma) \int_{t-\sigma}^{t} f(s, x(s)) d s,
$$

where $f(t,$.$) is nondecreasing, was studied by Ait Dads and Ezzinbi in [1]. In [2],$ Ait Dads, Cieutat and Lhachimi considered the existence of a positive solution for (3) via Hilbert's projective metric on a subset of a space of continuous and bounded functions, where $f(t,$.$) is not necessarily monotone.$

On the other hand, since the authors in [9] proposed the following system

$$
\begin{aligned}
& x(t)=\int_{t-\tau_{1}}^{t} f(s, x(s), y(s)) d s \\
& y(t)=\int_{t-\tau_{2}}^{t} g(s, x(s), y(s)) d s
\end{aligned}
$$


which was considered as a model to explain the population growth of two species in a periodic environment, there have been many papers concerned with the existence of positive periodic solutions for (4) and other forms

$$
\begin{aligned}
& x(t)=\int_{0}^{\tau_{1}(t)} f(t, s, x(t-s-l), y(t-s-l)) d s \\
& y(t)=\int_{0}^{\tau_{2}(t)} g(t, s, x(t-s-l), y(t-s-l)) d s
\end{aligned}
$$

In $[7,16,20]$, the authors studied the existence of positive periodic solutions for the above systems by using the method of upper and lower solutions, where the hypothesis of monotonicity is imposed on the functions $f$ and $g$. In $[6,17]$, the authors used the topological method to prove the existence of positive periodic solutions for the above system, where the monotonocity is not imposed. Recently, in [18] we show the existence and uniqueness of positive almost periodic solution of (4) in the case of variant delays, and where we suppose that the functions $f$ and $g$ admit a suitable decomposition and are monotone with respect to $x$ and $y$.

In the present work, we show the existence and uniqueness of positive weighted pseudo almost automorphic solution for the system (1), which is more general than (4), via the Thompson's part metric and by using the Banach's fixed point. We extend some existing results even in the scalar cases $[2,19]$ and generalize others in the cases of systems [18]. We are interested in the "coexistence states", that is the existence of weighted pseudo almost automorphic solution $(\mathrm{x}, \mathrm{y})$ with both components positive. Therefore, due to the biological point of view, the condition $f(t, 0, y)=g(t, x, 0)=0, \forall(t, x, y) \in \mathbb{R} \times \mathbb{R}^{+} \times \mathbb{R}^{+}$ is completely coherent because, if the number of individuals of the species $x$, or $y$, is zero at some time, then the number of new births of this species must be zero. Also, this implies that $(0,0)$ is always a solution of (1).

The paper is organized as follows. In Section 2, we collect some well known definitions and results. Namely, the notions of almost autumorphy, weighted pseudo almost automorphy and the Thompson's part metric on the cones are presented. Also, we extend the notion of Thompson's part metric to the case of the product of two parts. Section 3 is devoted to prove the existence and uniqueness theorem of positive weighted pseudo almost automorphic solution. In Section 4, we give an example to illustrate our results. 


\section{Preliminaries}

Throughout this paper, we denote by $\mathbb{R}$ the set of real numbers, by $\mathbb{R}^{+}$the set of nonnegative real numbers, by $\Omega$ a closed subset in $\mathbb{R} \times \mathbb{R}$ and by $C(X)$, where $X$ is a metric space, the space of continuous functions defined on $X$ with values in $\mathbb{R}$. For $f \in C(\mathbb{R})$ and $\beta \in \mathbb{R}$, the translation of $f$ is the function $\tau_{\beta} f(t)=f(t-\beta), t \in \mathbb{R}$.

Definition 1 ([13]). A continuous function $f: \mathbb{R} \longrightarrow \mathbb{R}$ is called almost automorphic, if for every sequence of real numbers $\left(S_{m}^{\prime}\right)_{m}$ there exists a subsequence $\left(S_{n}\right)_{n}$ such that

$$
\lim _{m \rightarrow+\infty} \lim _{n \rightarrow+\infty} f\left(t+S_{n}-S_{m}\right)=f(t), \forall t \in \mathbb{R}
$$

This limit means that

$$
g(t)=\lim _{n \rightarrow+\infty} f\left(t+S_{n}\right)
$$

is well defined for each $t \in \mathbb{R}$ and

$$
f(t)=\lim _{n \rightarrow+\infty} g\left(t-S_{n}\right), \forall t \in \mathbb{R} .
$$

The collection of all such functions will be denoted by $A A(\mathbb{R})$.

Notice that some fundamental properties of almost periodic functions are not verified by the almost automorphic functions, as example the property of uniform continuity. A well known example of almost automorphic function not almost periodic is

$$
f(t)=\cos \frac{1}{2+\sin t+\sin \sqrt{2} t} .
$$

Lemma 2 ([13]). Assume that $f, g \in A A(\mathbb{R})$ and $\lambda$ is any scalar. Then the following hold true:

i) $f+g, f . g, \lambda f, f_{\tau}(t)=f(t+\tau), \tilde{f}(t)=f(-t)$ are almost automorphic.

ii) The range $R_{f}=\{f(t): t \in \mathbb{R}\}$ is precompact in $\mathbb{R}$, and so $f$ is bounded.

iii) If $\left\{f_{n}\right\}$ is a sequence of almost automorphic functions and $f_{n} \rightarrow f$ uniformly on $\mathbb{R}$, then $f$ is almost automorphic. 
iii) Equipped with the sup norm

$$
\|f\|=\sup _{t \in \mathbb{R}}|f(t)|
$$

$A A(\mathbb{R})$ turns out to be a Banach space.

Definition 3 ([13]). A continuous function $f: \mathbb{R} \times \Omega \longrightarrow \mathbb{R}$ is called almost automorphic in $t$ uniformly for $(x, y)$ in compact subset of $\Omega$ if for every compact subset $K$ of $\Omega$ and every real sequence $\left(S_{m}\right)_{m}$, there exists a subsequence $\left(S_{n}\right)_{n}$ such that

$$
g(t, x, y)=\lim _{n \rightarrow+\infty} f\left(t+S_{n}, x, y\right)
$$

is well defined for each $t \in \mathbb{R},(x, y) \in K$ and

$$
f(t, x, y)=\lim _{n \rightarrow+\infty} g\left(t-S_{n}, x, y\right), \forall t \in \mathbb{R} .
$$

The collection of all such functions will be denoted by $A A(\mathbb{R} \times \Omega)$.

Let $U$ denote the collection of all functions (weights) $\rho: \mathbb{R} \longrightarrow(0,+\infty)$ which are locally integrable over $\mathbb{R}$ such that $\rho(t)>0$ for almost each $t \in \mathbb{R}$. For $\rho \in U$ and $r>0$, we set

$$
m(r, \rho)=\int_{-r}^{r} \rho(t) d t
$$

Throughout this paper, the set of weights $U_{\infty}$ stands for

$$
U_{\infty}=\left\{\rho \in U: \lim _{r \rightarrow+\infty} m(r, \rho)=\infty\right\} .
$$

Obviously, $U_{\infty} \subset U$, with strict inclusions.

Let $\rho \in U_{\infty}$. Set

$$
P A A_{0}(\mathbb{R}, \rho)=\left\{f \in B C(\mathbb{R}): \lim _{r \rightarrow+\infty} \frac{1}{m(r, \rho)} \int_{-r}^{r}|f(t)| \rho(t) d t=0\right\} .
$$

In the same way, we define $P A A_{0}\left(\mathbb{R} \times \mathbb{R}^{+} \times \mathbb{R}^{+}, \rho\right)$ as the collection of continuous functions $f$ defined on $\mathbb{R} \times \mathbb{R}^{+} \times \mathbb{R}^{+}$such that $f(., x, y)$ is bounded for each $(x, y) \in \mathbb{R}^{+} \times \mathbb{R}^{+}$and

$$
\lim _{r \rightarrow+\infty} \frac{1}{m(r, \rho)} \int_{-r}^{r}|f(t, x, y)| \rho(t) d t=0
$$

uniformly in $(x, y) \in \mathbb{R}^{+} \times \mathbb{R}^{+}$. 
Definition $4([5])$. Let $\rho \in U_{\infty}$. A function $f \in B C(\mathbb{R})$ is called weighted pseudo almost automorphic (or $\rho$-pseudo almost automorphic), if it can be expressed as $f=f^{a a}+f^{e}$, where $f^{a a} \in A A(\mathbb{R})$ and $f^{e} \in P A A_{0}(\mathbb{R}, \rho)$. The collection of such functions is denoted by $W P A A(\mathbb{R}, \rho)$.

The functions $f^{a a}$ and $f^{e}$ appearing in definition above are respectively called the almost periodic and the weighted ergodic perturbation components of $f$.

Example 5. Let $\rho(t)=e^{t}$ for each $t \in \mathbb{R}$. Clearly $\rho \in U_{\infty}$ and the function

$$
f(t)=\cos \frac{1}{2+\sin t+\sin \sqrt{2} t}+e^{-t}
$$

is in $W P A A(\mathbb{R}, \rho)$, since

$$
\lim _{r \rightarrow+\infty} \frac{1}{m(r, \rho)} \int_{-r}^{r} e^{-t} e^{t} d t=0 .
$$

Lemma 6 ([5, 14]). Let $\rho \in U_{\infty}$.

(i) $W P A A(\mathbb{R}, \rho)$ equipped with the sup norm is a Banach space.

(ii) If $f=f^{a a}+f^{e} \in W P A A(\mathbb{R}, \rho)$ with $f^{a a} \in A A(\mathbb{R})$ and $f^{e} \in P A A_{0}(\mathbb{R}, \rho)$, then $f^{a a}(\mathbb{R}) \subset \overline{f(\mathbb{R})}$.

(iii) If $f \in B C(\mathbb{R})$, then $f \in P A A_{0}(\mathbb{R}, \rho)$ if and only if for every $\varepsilon>0$

$$
\lim _{r \rightarrow+\infty} \frac{1}{m(r, \rho)} \int_{M_{r, \varepsilon}(f)} \rho(t) d t=0
$$

where $M_{r, \varepsilon}(f)=\{t \in[-r, r]: \| f(t) \geq \varepsilon\}$.

(iv) If we consider that $\rho \equiv 1$, then we obtain the standard spaces $P A A(\mathbb{R})$ and $P A A\left(\mathbb{R} \times \mathbb{R}^{+} \times \mathbb{R}^{+}\right)$.

Lemma 7. Let us fix $\rho \in U_{\infty}$.

1) Following the same reasoning as in the proof of [4] it follows that If $f, g \in$ $W P A A(\mathbb{R}, \rho)$, then $f . g \in W P A A(\mathbb{R}, \rho)$ 
2) We know from Agarwal et al. [3] that if the limits

$$
\limsup _{t \rightarrow \infty} \frac{\rho(t+\tau)}{\rho(t)}<\infty \text { and } \limsup _{t \rightarrow \infty} \frac{m(r+\tau, \rho)}{m(r, \rho)}<\infty
$$

exist for each $\tau \in \mathbb{R}$. Then the space $P A A_{0}(\mathbb{R}, \rho)$ is translation invariant, that is, if $f \in W P A A(\mathbb{R}, \rho)$, then $f(.-\tau) \in W P A A(\mathbb{R}, \rho)$ where $\tau$ is a fxed constant.

Definition 8 ([5]). A function $f \in B C\left(\mathbb{R} \times \mathbb{R}^{+} \times \mathbb{R}^{+}\right)$is called weighted pseudo almost automorphic (or $\rho$-pseudo almost automorphic ) if it can be expressed as $f=f^{a a}+f^{e}$, where $f^{a a} \in A A\left(\mathbb{R} \times \mathbb{R}^{+} \times \mathbb{R}^{+}\right)$and $f^{e} \in P A A_{0}(\mathbb{R} \times$ $\left.\mathbb{R}^{+} \times \mathbb{R}^{+}, \rho\right)$. The collection of such functions is denoted by $W P A A\left(\mathbb{R} \times \mathbb{R}^{+} \times\right.$ $\left.\mathbb{R}^{+}, \rho\right)$.

Theorem $9([5,12])$. Fix $\rho \in U_{\infty}$. Let $x, y \in W P A A(\mathbb{R}, \rho)$ and $f=$ $f^{a a}+f^{e} \in W P A A\left(\mathbb{R} \times \mathbb{R}^{+} \times \mathbb{R}^{+}, \rho\right)$. Assume both $f$ and $f^{a a}$ are uniformly continuous in any bounded subset $K \in \mathbb{R}^{+} \times \mathbb{R}^{+}$uniformly in $t \in \mathbb{R}$. Then, $f(., x(),. y().) \in W P A A(\mathbb{R}, \rho)$.

Corollary 10 ([14]). Fix $\rho \in U_{\infty}$. Let $x, y \in W P A A(\mathbb{R}, \rho)$ and $f=$ $f^{a a}+f^{e} \in W P A A\left(\mathbb{R} \times \mathbb{R}^{+} \times \mathbb{R}^{+}, \rho\right)$. Assume both $f$ and $f^{a a}$ are lipschitzian in $(x, y) \in \mathbb{R}^{+} \times \mathbb{R}^{+}$uniformly in $t \in \mathbb{R}$. Then, $f(., x(),. y().) \in W P A A(\mathbb{R}, \rho)$.

Lemma 11. Let $\rho \in U_{\infty}$ and $f \in W P A A(\mathbb{R}, \rho), \sigma \in W P A A(\mathbb{R}, \rho)$ are nonnegative functions. Suppose that $P A A_{0}(\mathbb{R}, \rho)$ is translation invariant. Then

$$
F(t)=\int_{t-\sigma(t)}^{t} f(s) d s \in W P A A(\mathbb{R}, \rho) .
$$

Proof. Since $f, \sigma \in W P A A(\mathbb{R}, \rho)$, there exist $h, \tau \in A A(\mathbb{R})$ and $\varphi, \psi \in$ $P A A_{0}(\mathbb{R}, \rho)$ such that $f=h+\varphi$ and $\sigma=\tau+\psi$. It follows that

$$
\begin{aligned}
\int_{t-\sigma(t)}^{t} f(s) d s & =\int_{t-(\tau(t)+\psi(t))}^{t-\tau(t)} f(s) d s+\int_{t-\tau(t)}^{t} h(s) d s+\int_{t-\tau(t)}^{t} \varphi(s) d s \\
& =H(t)+\Phi(t),
\end{aligned}
$$

where

$$
H(t)=\int_{t-\tau(t)}^{t} h(s) d s \text { and } \Phi(t)=\int_{t-(\tau(t)+\psi(t))}^{t-\tau(t)} f(s) d s+\int_{t-\tau(t)}^{t} \varphi(s) d s .
$$


It is well known that $H(t)=\int_{t-\tau(t)}^{t} h(s) d s \in A A(\mathbb{R})$ (see [11]). On the other hand,

$$
\begin{aligned}
& \frac{1}{m(r, \rho)} \int_{-r}^{r}\left|\int_{t-\tau(t)}^{t} \varphi(s) d s\right| \rho(t) d t \leq \frac{1}{m(r, \rho)} \int_{-r}^{r}\left|\int_{-\infty}^{t} \varphi(s) d s\right| \rho(t) d t \\
& =\frac{1}{m(r, \rho)} \int_{-r}^{r}\left|\int_{0}^{+\infty} \varphi(t-s) d s\right| \rho(t) d t \\
& \leq \int_{0}^{+\infty}\left(\frac{1}{m(r, \rho)} \int_{-r}^{r}|\varphi(t-s)| \rho(t) d t\right) d s \\
& =\int_{0}^{+\infty} K_{r}(s) d s,
\end{aligned}
$$

where $K_{r}(s)=\frac{1}{m(r, \rho)} \int_{-r}^{r}|\varphi(t-s)| \rho(t) d t$.

By the definitions of $K_{r}$ and $m(r, \rho)$, one can see that $K_{r}$ is bounded and $K_{r} \geq 0$. In addition, since $P A A_{0}(\mathbb{R}, \rho)$ is translation invariant and $\varphi \in$ $P A A_{0}(\mathbb{R}, \rho)$, we see that $\varphi(.-s) \in P A A_{0}(\mathbb{R}, \rho)$, that is, $\lim _{r \rightarrow+\infty} K_{r}(s)=0$. Thus, by the Lebegue dominated convergence theorem, we obtain

$$
\lim _{r \rightarrow+\infty} \frac{1}{m(r, \rho)} \int_{-r}^{r}\left|\int_{t-\tau(t)}^{t} \varphi(s) d s\right| \rho(t) d t=0 .
$$

Also, we have

$$
\frac{1}{m(r, \rho)} \int_{-r}^{r}\left|\int_{t-(\tau(t)+\psi(t))}^{t-\tau(t)} f(s) d s\right| \rho(t) d t \leq\|f\|_{\infty} \frac{1}{m(r, \rho)} \int_{-r}^{r}|\psi(t)| \rho(t) d t .
$$

And since $\frac{1}{m(r, \rho)} \int_{-r}^{r}|\psi(t)| \rho(t) d t \underset{r \longrightarrow \infty}{\longrightarrow} 0$, we have

$$
\lim _{r \rightarrow \infty} \frac{1}{m(r, \rho)} \int_{-r}^{r}\left|\int_{t-(\tau(t)+\psi(t))}^{t-\tau(t)} f(s) d s\right| \rho(t) d t=0 .
$$

Definition 12. Let $X$ be a real Banach space. A close convex set $P$ is called a convex cone, if the following conditions are satisfied

1. If $x \in P$, then $\lambda x \in P$ for any $\lambda \in \mathbb{R}^{+}$;

2. If $x \in P$ and $-x \in P$, then $x=0$. 
A cone $P$ induces a partial ordering $\leq$ in $X$ by $x \leq y$ if, and only if, $y-x \in P$. A cone $P$ is called normal if there exists a constant $N>0$ such that $0 \leq x \leq y$ implies $\|x\| \leq N\|y\|$, where $\|$.$\| is the norm on X$. We denote by $\stackrel{\circ}{P}$ the interior set of $P$. A cone $P$ is called a solid cone if $\stackrel{\circ}{P} \neq \emptyset$.

Recall that for all $x, y \in \stackrel{\circ}{P}$ there exist numbers $\lambda, \mu \in(0,+\infty)$ such that $x \leq \lambda y$ and $y \leq \mu x$. Moreover, if we consider $\alpha=\inf \{\lambda>0: x \leq \lambda y\}$ and $\beta=\inf \{\mu>0: y \leq \mu x\}$ then, since $P$ is closed, $x \leq \alpha y$ and $y \leq \beta x$. Define the Thompson's part metric $d(x, y)$ by

$$
d(x, y)=\ln (\max \{\alpha, \beta\}) .
$$

Lemma $13([19])$. $d$ gives a metric on $\stackrel{\circ}{P}$ and $(\stackrel{\circ}{P}, d)$ is a complete metric space.

Now, let $(x, y),\left(x^{\prime}, y^{\prime}\right) \in \stackrel{\circ}{P} \times \stackrel{\circ}{P}$. Set $\alpha^{*}=\inf \left\{\lambda>0: x \leq \lambda x^{\prime}\right.$ and $\left.y \leq \lambda y^{\prime}\right\}$, $\beta^{*}=\inf \left\{\lambda>0: x^{\prime} \leq \lambda x\right.$ and $\left.y^{\prime} \leq \lambda y\right\}$. Then, $x \leq \alpha^{*} x^{\prime}, y \leq \alpha^{*} y^{\prime}, x^{\prime} \leq \beta^{*} x$ and $y^{\prime} \leq \beta^{*} y$. We define $\delta\left((x, y),\left(x^{\prime}, y^{\prime}\right)\right)$ by

$$
\delta\left((x, y),\left(x^{\prime}, y^{\prime}\right)\right)=\ln \left(\max \left\{\alpha^{*}, \beta^{*}\right\}\right) .
$$

Lemma 14. $\delta$ gives a metric on $\stackrel{\circ}{P} \times \stackrel{\circ}{P}$ and $(\stackrel{\circ}{P} \times \stackrel{\circ}{P}, \delta)$ is a complete metric space.

Proof. From the definition of $\delta$, it is evident that

$$
\delta\left((x, y),\left(x^{\prime}, y^{\prime}\right)\right)=\delta\left(\left(x^{\prime}, y^{\prime}\right),(x, y)\right)
$$

If $(x, y) \neq\left(x^{\prime}, y^{\prime}\right)$ then $x \neq x^{\prime}$ or $y \neq y^{\prime}$, and by remarking that $\delta\left((x, y),\left(x^{\prime}, y^{\prime}\right)\right) \geq$ $\max \left\{d\left(x, x^{\prime}\right), d\left(y, y^{\prime}\right)\right\}$, we get $\delta\left((x, y),\left(x^{\prime}, y^{\prime}\right)\right) \geq 0$. Also, if $\delta\left((x, y),\left(x^{\prime}, y^{\prime}\right)\right)=0$ then $d\left(x, x^{\prime}\right)=d\left(y, y^{\prime}\right)=0$, hence $(x, y)=\left(x^{\prime}, y^{\prime}\right)$.

Let $(x, y),\left(x^{\prime}, y^{\prime}\right),(u, v) \in \stackrel{\circ}{P} \times \stackrel{\circ}{P}$ be such that

$$
\begin{array}{ll}
x \leq \alpha_{1}^{*} x^{\prime} \text { and } y \leq \alpha_{1}^{*} y^{\prime}, & x^{\prime} \leq \beta_{1}^{*} x \text { and } y^{\prime} \leq \beta_{1}^{*} y, \\
x \leq \alpha_{2}^{*} u \text { and } y \leq \alpha_{2}^{*} v, & u \leq \beta_{2}^{*} x \text { and } v \leq \beta_{2}^{*} y, \\
x^{\prime} \leq \alpha_{3}^{*} u \text { and } y^{\prime} \leq \alpha_{3}^{*} v, & u \leq \beta_{3}^{*} x^{\prime} \text { and } v \leq \beta_{3}^{*} y^{\prime},
\end{array}
$$


where $\alpha_{i}^{*}, \beta_{i}^{*}(i=1,2,3)$ are the infimum of all numbers satisfying the respective inequalities. If $\alpha_{1}^{*} \geq \beta_{1}^{*}$, we have

$$
\begin{aligned}
\delta\left((x, y),\left(x^{\prime}, y^{\prime}\right)\right)=\ln \alpha_{1}^{*} & \leq \ln \alpha_{2}^{*} \beta_{3}^{*}=\ln \alpha_{2}^{*}+\ln \beta_{3}^{*} \\
& \leq \ln \left(\max \left\{\alpha_{2}^{*}, \beta_{2}^{*}\right\}\right)+\ln \left(\max \left\{\alpha_{3}^{*}, \beta_{3}^{*}\right\}\right) \\
& \leq \delta((x, y),(u, v))+\delta\left(\left(x^{\prime}, y^{\prime}\right),(u, v)\right) .
\end{aligned}
$$

Similarly if $\beta_{1}^{*} \geq \alpha_{1}^{*}$. Finally, to prove that $\stackrel{\circ}{P} \times \stackrel{\circ}{P}$ is complete with respect to $\delta$, we consider $\left\{\left(x_{n}, y_{n}\right)\right\}$ a sequence in $\stackrel{\circ}{P} \times \stackrel{\circ}{P}$ which is Cauchy with respect to $\delta$. Then, since $\delta\left((x, y),\left(x^{\prime}, y^{\prime}\right)\right) \geq \max \left\{d\left(x, x^{\prime}\right), d\left(y, y^{\prime}\right)\right\}$ for each $(x, y),\left(x^{\prime}, y^{\prime}\right) \in$ $\stackrel{\circ}{P} \times \stackrel{\circ}{P},\left\{x_{n}\right\}$ and $\left\{y_{n}\right\}$ are sequences in $\stackrel{\circ}{P}$ which are Cauchy with respect to $d$, and since $(\stackrel{\circ}{P}, d)$ is complete, there exists $(x, y) \in \stackrel{\circ}{P} \times \stackrel{\circ}{P}$ such that $\left\{\left(x_{n}, y_{n}\right)\right\}$ converge to $(x, y)$.

To prove our result, we apply the following Banach's fixed point theorem.

Theorem 15 ([15], p.15). Let $(M, \delta)$ be a complete metric space and $T: M \longrightarrow M$ be a contraction mapping with contraction constant $k$. Then $T$ has a unique fixed point $x \in M$. Furthermore, if $y \in M$ is arbitrarily chosen, then the iterates $\left\{x_{n}\right\}_{n=0}^{\infty}$ given by

$$
\begin{gathered}
x_{0}=y \\
x_{n}=T\left(x_{n-1}\right), n \geq 1,
\end{gathered}
$$

have the property that $\lim _{n \rightarrow+\infty} x_{n}=x$.

\section{Main Result}

In this section, we prove the existence and uniqueness of a positive weighted pseudo almost automorphic solution for the system (1). Throughout the rest of this paper, we consider $\rho \in U_{\infty}$ such that $P A A_{0}(\mathbb{R}, \rho)$ is translation invariant. Denote by $P$ the following set

$$
P=\{x \in W P A A(\mathbb{R}, \rho): x(t) \geq 0, \forall t \in \mathbb{R}\} .
$$

It is easy to verify that $P$ is a normal and solid cone in $W P A A(\mathbb{R}, \rho)$ with

$$
\stackrel{\circ}{P}=\{x \in P: \exists \varepsilon>0 \text { such that } x(t)>\varepsilon, \forall t \in \mathbb{R}\} .
$$

We list some assumptions: 
$\left(\mathrm{H}_{1}\right) \gamma_{1}, \gamma_{2} \in W P A A(\mathbb{R}, \rho)$ are nonnegative functions verifying

$$
\sup _{t \in \mathbb{R}} \gamma_{i}(t)<1, i=1,2 .
$$

$\left(\mathrm{H}_{2}\right) f, g \in W P A A\left(\mathbb{R} \times \mathbb{R}^{+} \times \mathbb{R}^{+}, \rho\right)$ are nonnegative functions such that $f, g$, $f^{a a}, g^{a a}$ are uniformly continuous in any bounded subset $K \in \mathbb{R}^{+} \times \mathbb{R}^{+}$ uniformly in $s \in \mathbb{R}$ and $\sigma_{1}, \sigma_{2} \in W P A A(\mathbb{R}, \rho)$ are positive functions.

$\left(\mathrm{H}_{3}\right)$ There exist positive functions $\varphi_{1}, \varphi_{2}:(0,1) \times(0,+\infty) \times(0,+\infty) \rightarrow \mathbb{R}$, $\psi_{i}:(0,1) \times(0,1] \rightarrow \mathbb{R}$ and a constant $k \in[0,1)$ such that

$$
\inf _{r \leq x, y \leq r^{-1}} \varphi_{i}(\lambda, x, y)=\psi_{i}(\lambda, r)>\lambda^{k}(i=1,2),
$$

$\forall r \in(0,1], \forall \lambda \in(0,1)$ and

$$
\begin{gathered}
\left\{\begin{array} { l } 
{ \lambda x ^ { \prime } \leq x \leq \lambda ^ { - 1 } x ^ { \prime } } \\
{ \lambda y ^ { \prime } \leq y \leq \lambda ^ { - 1 } y ^ { \prime } }
\end{array} \Longrightarrow \left\{\begin{array}{l}
f(t, x, y) \geq \varphi_{1}\left(\lambda, x^{\prime}, y^{\prime}\right) f\left(t, x^{\prime}, y^{\prime}\right) \\
g(t, x, y) \geq \varphi_{2}\left(\lambda, x^{\prime}, y^{\prime}\right) g\left(t, x^{\prime}, y^{\prime}\right)
\end{array}\right.\right. \\
\forall x, x^{\prime}, y, y^{\prime} \in(0,+\infty), \forall(\lambda, t) \in(0,1) \times \mathbb{R} .
\end{gathered}
$$

Now, we are ready to present our results.

Theorem 16. Suppose that $\left(H_{1}\right)-\left(H_{3}\right)$ hold. In addition:

$\left(H_{4}\right)$ There exist continuous functions $a, b: \mathbb{R} \rightarrow \mathbb{R}$ and numbers $p, q \in[0,+\infty)$ such that

$$
\liminf _{(x, y) \rightarrow\left(0^{+}, 0^{+}\right)} \frac{f(t, x, y)}{x^{p}}=a(t), \liminf _{(x, y) \rightarrow\left(0^{+}, 0^{+}\right)} \frac{g(t, x, y)}{y^{q}}=b(t)
$$

uniformly in $t \in \mathbb{R}$. Moreover,

$$
\min _{t \in \mathbb{R}} \int_{t-\sigma_{1}(t)}^{t} a(s) d s>0 \text { and } \min _{t \in \mathbb{R}} \int_{t-\sigma_{2}(t)}^{t} b(s) d s>0 .
$$

Then system (1) has a unique solution in $\stackrel{\circ}{P} \times \stackrel{\circ}{P}$.

Proof. By Lemma 7 and the fact that $P A A_{0}(\mathbb{R}, \rho)$ is translation invariant, we have $\left(I d-\gamma_{i} \tau_{\beta_{i}}\right)(x)$ is belongs to $W P A A(\mathbb{R}, \rho)$ for all $x \in W P A A(\mathbb{R}, \rho)$, where $\left(\gamma_{i} \tau_{\beta_{i}} x\right)(t)=\gamma_{i}(t) x\left(t-\beta_{i}\right)$ and $i=1$, 2. In addition, from $\left(\mathrm{H}_{1}\right)$, one sees 
that the operators $I d-\gamma_{1} \tau_{\beta_{1}}$ and $I d-\gamma_{2} \tau_{\beta_{2}}$ are invertible on $W P A A(\mathbb{R}, \rho)$ with inverses $L_{1}$ and $L_{2}$ giving by

$$
L_{i}=I d+\sum_{n \geq 1}\left(\prod_{k=0}^{n-1} \tau_{k \beta_{i}} \gamma_{i}\right) \tau_{n \beta_{i}}, i=1,2,
$$

where $\left(\left(\tau_{k \beta_{i}} \gamma_{i}\right) \tau_{n \beta_{i}} x\right)(t)=\gamma_{i}\left(t-k \beta_{i}\right) x\left(t-n \beta_{i}\right)$ for $x \in W P A A(\mathbb{R}, \rho)$ and $t \in \mathbb{R}$. Also, we have for all $x, y \in W P A A(\mathbb{R}, \rho)$

$$
x \leq y \text { implies } L_{1} x \leq L_{1} y \text { and } L_{2} x \leq L_{2} y .
$$

By setting $u=\left(I d-\gamma_{1} \tau_{\beta_{1}}\right) x$ and $v=\left(I d-\gamma_{2} \tau_{\beta_{2}}\right) y$, we obtain from $\left(\mathrm{H}_{2}\right)$, Theorem 9 and Lemma 11 that the system (1) is equivalent to

$$
\begin{aligned}
& u(t)=\int_{t-\sigma_{1}(t)}^{t} f\left(s,\left(L_{1} u\right)(s),\left(L_{2} v\right)(s)\right) d s \\
& v(t)=\int_{t-\sigma_{2}(t)}^{t} g\left(s,\left(L_{1} u\right)(s),\left(L_{2} v\right)(s)\right) d s
\end{aligned}
$$

We claim that $(x, y) \in \stackrel{\circ}{P} \times \stackrel{\circ}{P}$ is a solution of (1) if, and only if $(u, v) \in \stackrel{\circ}{P} \times \stackrel{\circ}{P}$ is a solution of (8). In fact, it suffices to prove that $(x, y) \in \stackrel{\circ}{P} \times \stackrel{\circ}{P}$ if, and only if $(u, v) \in \stackrel{\circ}{P} \times \stackrel{\circ}{P}$.

By $\left(\mathrm{H}_{4}\right)$, for every $\varepsilon>0$ satisfying $\min _{t \in \mathbb{R}} \int_{t-\sigma_{1}(t)}^{t}(a(s)-\varepsilon) d s>0$ there exists $\eta \in(0,1)$ such that

$$
f(t, x, y) \geq(a(s)-\varepsilon) x^{p}, \quad \forall x \leq \eta, \forall y \leq \eta .
$$

Let $(x, y) \in \stackrel{\circ}{P} \times \stackrel{\circ}{P}$ be a solution of $(1)$. Then, there exists $\lambda \in(0,1)$ verifying $\lambda \eta \leq x(s) \leq \lambda^{-1} \eta$ and $\lambda \eta \leq y(s) \leq \lambda^{-1} \eta, \forall s \in \mathbb{R}$. It follows

$$
\begin{aligned}
u(t)=x(t)-\gamma_{1}(t) x\left(t-\beta_{1}\right) & =\int_{t-\sigma_{1}(t)}^{t} f(s, x(s), y(s)) d s \\
& \geq \int_{t-\sigma_{1}(t)}^{t} \varphi_{1}(\lambda, \eta, \eta) f(s, \eta, \eta) d s \\
& \geq \lambda^{k} \eta^{p} \int_{t-\sigma_{1}(t)}^{t}(a(s)-\varepsilon) d s>0 .
\end{aligned}
$$

Hence, $\min _{t \in \mathbb{R}} u(t)>0$. Similarly we obtain $\min _{t \in \mathbb{R}} v(t)>0$. Conversely, if $(u, v) \in$ $\stackrel{\circ}{P} \times \stackrel{\circ}{P}$, then by $(7)$, we have $(x, y) \in \stackrel{\circ}{P} \times \stackrel{\circ}{P}$. In the sequel, we set 


$$
\begin{aligned}
& T_{1}(x, y)(t)=\int_{t-\sigma_{1}(t)}^{t} f\left(s,\left(L_{1} x\right)(s),\left(L_{2} y\right)(s)\right) d s \\
& T_{2}(x, y)(t)=\int_{t-\sigma_{2}(t)}^{t} g\left(s,\left(L_{1} x\right)(s),\left(L_{2} y\right)(s)\right) d s
\end{aligned}
$$

and

$$
T(x, y)(t)=\left(T_{1}(x, y)(t), T_{2}(x, y)(t)\right) .
$$

Then $(x, y)$ is a solution of (1) if, and only if, $T(x, y)=(x, y)$.

We prove that $T: \stackrel{\circ}{P} \times \stackrel{\circ}{P} \longrightarrow \stackrel{\circ}{P} \times \stackrel{\circ}{P}$. Let $\varepsilon>0$ satisfying

$$
\min _{t \in \mathbb{R}} \int_{t-\sigma_{1}(t)}^{t}(a(s)-\varepsilon) d s>0 .
$$

By $\left(\mathrm{H}_{4}\right)$, there exists $\eta \in(0,1)$ such that

$$
f(t, x, y) \geq(a(s)-\varepsilon) x^{p}, \forall x \leq \eta, \forall y \leq \eta
$$

For $(x, y) \in \stackrel{\circ}{P} \times \stackrel{\circ}{P}$, choose $\lambda \in(0,1)$ verifying $\lambda \eta \leq\left(L_{1} x\right)(s) \leq \lambda^{-1} \eta$ and $\lambda \eta \leq\left(L_{2} y\right)(s) \leq \lambda^{-1} \eta, \forall s \in \mathbb{R}$. Then, we have for each $s \in \mathbb{R}$

$$
\begin{aligned}
f\left(s,\left(L_{1} x\right)(s),\left(L_{2} y\right)(s)\right) & \geq \varphi_{1}(\lambda, \eta, \eta) f(s, \eta, \eta) \\
& \geq \lambda^{k}(a(s)-\varepsilon) \eta^{p} .
\end{aligned}
$$

It follows

$$
T_{1}(x, y)(t) \geq \lambda^{k} \eta^{p} \min _{t \in \mathbb{R}} \int_{t-\sigma_{1}(t)}^{t}(a(s)-\varepsilon) d s>0 .
$$

Thus, $T_{1}(x, y) \in \stackrel{\circ}{P}$. Similarly, we have $T_{2}(x, y) \in \stackrel{\circ}{P}$.

Consider $(x, y),\left(x^{\prime}, y^{\prime}\right) \in \stackrel{\circ}{P} \times \stackrel{\circ}{P}$ and $\lambda=\left[\max \left(\alpha^{*}, \beta^{*}\right)\right]^{-1}$, where $\alpha^{*}=$ $\inf \left\{\alpha>0: x \leq \alpha x^{\prime}\right.$ and $\left.y \leq \alpha y^{\prime}\right\}, \beta^{*}=\inf \left\{\beta>0: x^{\prime} \leq \beta x\right.$ and $\left.y^{\prime} \leq \beta y\right\}$. Then

$$
\lambda x^{\prime} \leq x \leq \lambda^{-1} x^{\prime}, \lambda y^{\prime} \leq y \leq \lambda^{-1} y^{\prime} \text { and } \delta\left((x, y),\left(x^{\prime}, y^{\prime}\right)\right)=\ln \left(\lambda^{-1}\right)
$$

From (7), we have

$$
\lambda L_{1} x^{\prime} \leq L_{1} x \leq \lambda^{-1} L_{1} x^{\prime} \text { and } \lambda L_{2} y^{\prime} \leq L_{2} y \leq \lambda^{-1} L_{2} y^{\prime} .
$$

It follows 


$$
\begin{aligned}
& f\left(s,\left(L_{1} x\right)(s),\left(L_{2} y\right)(s)\right) \\
& \geq \varphi_{1}\left(\lambda,\left(L_{1} x^{\prime}\right)(s),\left(L_{2} y^{\prime}\right)(s)\right) f\left(s,\left(L_{1} x^{\prime}\right)(s),\left(L_{2} y^{\prime}\right)(s)\right) \\
& \geq \psi_{1}(\lambda, r) f\left(s,\left(L_{1} x^{\prime}\right)(s),\left(L_{2} y^{\prime}\right)(s)\right),
\end{aligned}
$$

where $r \in(0,1]$ verifying $r \leq\left(L_{1} x^{\prime}\right)(s) \leq r^{-1}$ and $r \leq\left(L_{2} y^{\prime}\right)(s) \leq r^{-1}$ for every $s \in \mathbb{R}$. Thus,

$$
\psi_{1}(\lambda, r) T_{1}\left(x^{\prime}, y^{\prime}\right)(t) \leq T_{1}(x, y)(t), \forall t \in \mathbb{R} .
$$

Also, from (9) we have

$$
\lambda L_{1} x \leq L_{1} x^{\prime} \leq \lambda^{-1} L_{1} x \text { and } \lambda L_{2} y \leq L_{2} y^{\prime} \leq \lambda^{-1} L_{2} y,
$$

and similarly, we obtain

$$
T_{1}(x, y)(t) \leq\left[\psi_{1}(\lambda, r)\right]^{-1} T_{1}\left(x^{\prime}, y^{\prime}\right)(t), \forall t \in \mathbb{R}
$$

(think that $r$ satisfies also $r \leq\left(L_{1} x\right)(s) \leq r^{-1}$ and $r \leq\left(L_{2} y\right)(s) \leq r^{-1}$ for every $s \in \mathbb{R})$. Therefore

$$
\psi_{1}(\lambda, r) T_{1}\left(x^{\prime}, y^{\prime}\right)(t) \leq T_{1}(x, y)(t) \leq\left[\psi_{1}(\lambda, r)\right]^{-1} T_{1}\left(x^{\prime}, y^{\prime}\right)(t), \forall t \in \mathbb{R} .
$$

Analogously, we obtain

$$
\psi_{2}(\lambda, r) T_{2}\left(x^{\prime}, y^{\prime}\right)(t) \leq T_{2}(x, y)(t) \leq\left[\psi_{2}(\lambda, r)\right]^{-1} T_{2}\left(x^{\prime}, y^{\prime}\right)(t), \forall t \in \mathbb{R} .
$$

By setting $\psi(\lambda, r)=\min \left\{\psi_{1}(\lambda, r), \psi_{2}(\lambda, r)\right\}$, we have

$$
\delta\left(T(x, y), T\left(x^{\prime}, y^{\prime}\right)\right) \leq \ln \left[(\psi(\lambda, r))^{-1}\right] \leq k \delta\left((x, y),\left(x^{\prime}, y^{\prime}\right)\right) .
$$

This implies that $T: \stackrel{\circ}{P} \times \stackrel{\circ}{P} \longrightarrow \stackrel{\circ}{P} \times \stackrel{\circ}{P}$ is a contraction. By applying Theorem 15, $T$ has a unique fixed point in $\stackrel{\circ}{P} \times \stackrel{\circ}{P}$.

\section{Example}

Take $\beta_{1}=\beta_{2}=1, \sigma_{1}(t) \equiv \sigma_{1}, \sigma_{2}(t) \equiv \sigma_{2}$, with $\sigma_{1}, \sigma_{2}>0, \gamma_{1}(t)=\gamma_{2}(t) \equiv \frac{1}{2}$ and for $\beta \geq \alpha>0$, we consider

$$
a(t)=\sin \frac{1}{2+\cos t+\cos \sqrt{2} t}+e^{\alpha t}, \quad \rho(t)= \begin{cases}1 & \text { if } t<0 \\ e^{-\beta t} & \text { if } t \geq 0\end{cases}
$$


and

$$
\begin{aligned}
& f(t, x, y)=(a(t)+\rho(t)) \sqrt{\sqrt{x} \ln (1+\sqrt{x}) \ln (2+\sqrt{y})}, \\
& g(t, x, y)=(a(t)+\rho(t)) \sqrt{\sqrt{y} \ln (1+\sqrt{y}) \ln (2+\sqrt{x})} .
\end{aligned}
$$

Then

$$
\liminf _{(x, y) \rightarrow\left(0^{+}, 0^{+}\right)} \frac{f(t, x, y)}{\sqrt{x}}=\sqrt{\ln 2} a(t), \liminf _{(x, y) \rightarrow\left(0^{+}, 0^{+}\right)} \frac{g(t, x, y)}{\sqrt{y}}=\sqrt{\ln 2} b(t) .
$$

If $\lambda x^{\prime} \leq x \leq \lambda^{-1} x^{\prime}$ and $\lambda y^{\prime} \leq y \leq \lambda^{-1} y^{\prime}$, then

$$
\sqrt{\sqrt{x} \ln (1+\sqrt{x}) \ln (2+\sqrt{y})} \geq \sqrt{\sqrt{\lambda x^{\prime}} \ln \left(1+\sqrt{\lambda x^{\prime}}\right) \ln \left(2+\sqrt{\lambda y^{\prime}}\right)}
$$

By setting

$$
\varphi_{1}(\lambda, x, y)=\sqrt{\sqrt{\lambda} \frac{\ln (1+\sqrt{\lambda x}) \ln (2+\sqrt{\lambda y})}{\ln (1+\sqrt{x}) \ln (2+\sqrt{y})}},
$$

we obtain $f(t, x, y) \geq \varphi_{1}\left(\lambda, x^{\prime}, y^{\prime}\right) f\left(t, x^{\prime}, y^{\prime}\right)$. Similarly we have

$$
\varphi_{2}(\lambda, x, y)=\sqrt{\sqrt{\lambda} \frac{\ln (1+\sqrt{\lambda y}) \ln (2+\sqrt{\lambda x})}{\ln (1+\sqrt{y}) \ln (2+\sqrt{x})}}
$$

and $g(t, x, y) \geq \varphi_{2}\left(\lambda, x^{\prime}, y^{\prime}\right) g\left(t, x^{\prime}, y^{\prime}\right)$ with

$$
\varphi_{1}(\lambda, x, y)>\lambda^{\frac{3}{4}}, \varphi_{2}(\lambda, x, y)>\lambda^{\frac{3}{4}}
$$

$\forall x, y>0, \forall \lambda \in(0,1)$. Therefore, by Theorem 16, the following system of neutral integral equations

$$
\begin{aligned}
& x(t)=\frac{1}{2} x\left(t-\beta_{1}\right)+\int_{t-\sigma_{1}}^{t} a(s) \sqrt{\sqrt{x(s)} \ln (1+\sqrt{x(s)}) \ln (2+\sqrt{y(s)})} d s \\
& y(t)=\frac{1}{2} y\left(t-\beta_{2}\right)+\int_{t-\sigma_{2}}^{t} a(s) \sqrt{\sqrt{y(s)} \ln (1+\sqrt{y(s)}) \ln (2+\sqrt{x(s)})} d s
\end{aligned}
$$

has a unique weighted pseudo almost automorphic solution $(x, y)$ with $\inf _{t \in \mathbb{R}} x(t)>$ 0 and $\inf _{t \in \mathbb{R}} y(t)>0$. 


\section{References}

[1] E. Ait Dads, K. Ezzinbi, Almost periodic solution for some neutral nonlinear integral equation, Nonlinear Anal., 28 (1997), 1479-1489.

[2] E. Ait Dads, P. Cieutat and L. Lhachimi, Existence of positive almost periodic or ergodic solutions for some neutral nonlinear integral equations, J. of Differential and Integral Equations, 22 (2009), 1075-1096.

[3] R.P. Agarwal, T. Diagana, M.E. Hernandez, Weighted pseudo almost periodic solutions to some partial neutral functional differential equations, $J$. Nonlinear Convex Anal., 8 (2007), 397-415.

[4] B. Ammar, F. Chérif and M.A. Alimi, Existence and Uniqueness of pseudo almost periodic solutions of recurrent neural networks with time-varying coefficients and mixed delays, IEEE Trans. on Neural Networks and Learning Systems, 23 (2012), 109-118.

[5] J. Blot, G.M. Mophou, G.M. N’Guérékata and D. Pennequin, Weighted pseudo-almost automorphic functions and applications, Nonlinear Anal., 71 (2009), 903-909.

[6] A. Cañada, A. Zertiti, Fixed point theorems for systems of equations in ordered Banach spaces with applications to differential and integral equations, Nonlinear Analysis, 27, No 4 (1996), 397-411.

[7] A. Cañada, A. Zertiti, Systems of nonlinear delay integral equations modelling population growth in a periodic environment, Comment. Math. Univ. Carolinae, 35, No 4 (1994), 633-644.

[8] A. Cañada, A. Zertiti, Topological methods in the study of positive solutions for some nonlinear delay integral equations, Nonlinear Anal., 23, No 9 (1994), 1153-1165.

[9] K.L. Cooke, J.L. Kaplan, A periodic threshold theorem for epidemics and population growth, Math. Biosci., 31 (1976), 87-104.

[10] H.S. Ding, J. Liang, G.M. N'Guérékata, T.J. Xiao, Existence of positive almost automorphic solutions to neutral nonlinear integral equations, Nonlinear Analysis, 69 (2008), 1188-1199.

[11] H.S. Ding, W. Long, Positive almost automorphic solutions for some nonlinear delay integral equations, Electronic J. of Diff. Equations, 57 (2008), 1-8. 
[12] V. Kavitha, P. Z. Wang, R. Murugesu, Existence of weighted pseudo almost automorphic mild solution to fractional integro-differential equations, $J$. of Fractional Calculus and Applications, 4 (2013), 37-55.

[13] G.M. N'Guérékata, Topics in Almost Automorphy, Springer-Verlag, New York (2005).

[14] G.M. Mophou, Weighted pseudo almost automorphic mild solutions to semilinear fractional differential equations, Applied Mathematics and Computation, 217 (2011), 7579-7587.

[15] R.M. Brooks, K. Schmitt, The Contraction mapping principle and some applications, Electronic J. of Differential Equations, 09 (2009), 90 pp.

[16] A. Sadrati, A. Zertiti, A study of systems of nonlinear delay integral equations by using the method of upper and lower solutions, International $J$. of Math. and Computation, 17, No 4 (2012), 93-102.

[17] A. Sadrati, A. Zertiti, A topological methods for Existence and multiplicity of positive solutions for some systems of nonlinear delay integral equations, International J. of Mathematics and Statistics, 13, No 1 (2013), 47-55.

[18] A. Sadrati, A. Zertiti, Existence and uniqueness of positive almost periodic solution for systems of nonlinear delay ntegral equations, Electronic J. of Diff. Equations, 2015, No 116 (2015), 1-12.

[19] A.C. Thompson, On certain contraction mapping in a partially ordered vector space, Proc. Amer. Math. Soc., 14 (1963), 438-443.

[20] A. Zertiti, Method of upper and lower solutions applied to a nonlinear delay integral system, International J. of Mathematics and Statistics, 2 (2008), 115-120. 
